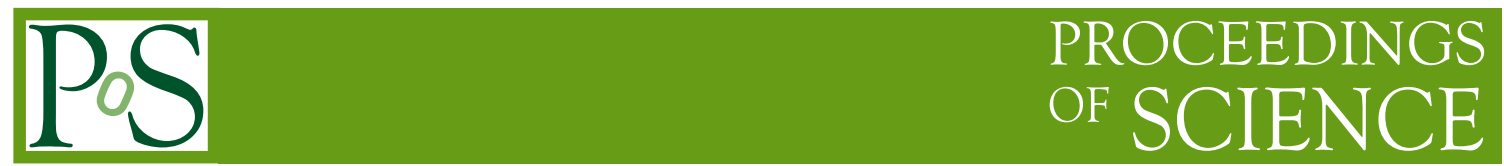

\title{
Renormalization-group blocking the fourth root of the staggered determinant
}

\author{
Yigal Shamir \\ School of Physics and Astronomy \\ Raymond and Beverly Sackler Faculty of Exact Sciences \\ Tel-Aviv University, Ramat Aviv, 69978 ISRAEL \\ E-mail: shamirepost.tau.ac.il
}

\begin{abstract}
Lattice QCD simulations with staggered fermions rely on the "fourth-root trick." The validity of this trick has been proved for free staggered fermions using renormalization-group block transformations. I review the elements of the construction and discuss how it might be generalized to the interacting case.
\end{abstract}

XXIIIrd International Symposium on Lattice Field Theory

25-30 July 2005

Trinity College, Dublin, Ireland 
1. The fourth-root trick. A staggered-fermion field $\chi(x)$ consists of a single fermionic degree of freedom per color per lattice site [1]. The sixteen variables (per color) residing in each $2^{4}$ hypercube of the euclidean lattice can be re-grouped into four quark fields in the continuum limit. Thus, a single staggered field could be used to simulate the up, down, strange and charm quarks, all at once, provided an appropriate mass matrix is chosen [2]. For various technical reasons, however, this is not the way staggered-fermion simulations are done.

The "rooted" Boltzmann weight used to generate the dynamical staggered-fermion configurations contains a factor of $\operatorname{det}^{1 / 4}\left(D_{s}+m\right)$ for each of the three light quarks, where $D_{s}$ is the massless, interacting, anti-hermitian, one-component, staggered-fermion operator. ${ }^{1}$ The raison d'etre behind the fourth-root trick is simple [3]. In the continuum limit, this staggered-fermion determinant will describe four equal-mass quarks that interact only through the exchange of gluons; hence, the determinant's fourth root is expected to account for a single quark with the same mass. The difficultly is that, for any finite lattice spacing $a$, the four quark species (or four "tastes," reserving the term "flavors" to the different-mass species) are entangled at short distances. There is no simple way to represent the rooted Boltzmann weight as a path integral with a local action. This raises the question whether the rooted staggered theory is consistent with the rules of local quantum field theory, and whether its continuum limit is in the same universality class as QCD. The unprecedented accuracy of staggered-fermions simulations has made this issue all the more imminent [ $[$ ].

In the free theory, the re-grouping alluded to in the first paragraph is explicitly given by [5]

$$
\psi_{\alpha i}(\tilde{x})=\sum_{r_{\mu}=0,1}\left(\gamma_{1}^{r_{1}} \gamma_{2}^{r_{2}} \gamma_{3}^{r_{3}} \gamma_{4}^{r_{4}}\right)_{\alpha i} \chi(2 \tilde{x}+r)
$$

Here $\alpha$ and $i$ are respectively Dirac and taste indices, that both run from 1 to 4 . The tasterepresentation variables $\psi_{\alpha i}$ live on a coarse lattice, with spacing $2 a$, whose sites are labeled by the coordinates $\tilde{x}$. Each site of the original, fine lattice has a unique representation as $x=2 \tilde{x}+r$, where $r_{\mu}=0,1$. In the taste basis the free (massless) staggered action is $S_{0}=\bar{\psi} D_{0} \psi$, where

$$
D_{0}=a^{-1} \sum_{\mu}\left(\left[\gamma_{\mu} \otimes I\right] i \sin \left(p_{\mu} a\right)+\left[\gamma_{5} \otimes \tau_{5} \tau_{\mu}\right]\left(1-\cos \left(p_{\mu} a\right)\right)\right.
$$

in momentum space. The two sets of Dirac matrices, $\gamma_{\mu}$ and $\tau_{\mu}=\gamma_{\mu}^{*}=\gamma_{\mu}^{T}$, act on the Dirac and taste indices respectively. $I$ is the identity matrix in taste space. The kinetic term-the first term on the right-hand side-is the naive discretization of the continuum $\not \supset$. The second term lifts the doublers at the $p \neq 0$ corners of the Brillouin zone (of the coarse lattice). We will refer to it as a skewed-because of the extra $\tau_{\mu}$ matrices-Wilson term.

As a start, there are two complementary ways to convince oneself that the fourth root "has to" work in the free theory. First, one can look for the four-fold degeneracy expected from the taste structure. In momentum space, the continuum limit of a free theory corresponds to $|p| a \rightarrow 0$. The kinetic term starts off as $\not p+O\left(a^{2} p^{3}\right)$, whereas the skewed Wilson term starts off as $O\left(a p^{2}\right)$; thus the skewed Wilson term drops out, and the expected four-fold degeneracy is obtained. Equivalently, one can examine the behavior of the propagator $G_{0}=D_{0}^{-1}$ at large space-time separations. The momentum-space representation of $G_{0}$ is easily worked out. The only singularity, occurring at $p \rightarrow 0$, has the same structure as in the continuum. The taste-breaking terms, originating in the

\footnotetext{
${ }^{1}$ In practice the one usually sets $m_{u}=m_{d}=m_{\text {light }}$ and simulates $\operatorname{det}^{1 / 2}\left(D_{s}+m_{\text {light }}\right) \operatorname{det}^{1 / 4}\left(D_{s}+m_{\text {strange }}\right)$.
} 
skewed Wilson term, do not produce a singularity in the propagator. As a result [6], the tastebreaking part of the coordinate-space free propagator vanishes exponentially with the separation, with an $O(1)$ decay rate in lattice units. We must keep in mind, though, that for momenta or separations which are $O(1)$ in lattice units, the skewed Wilson term does spoil the diagonal taste structure. Therefore we should not expect that merely taking the fourth root of the product of all the eigenvalues, small and large alike, will yield a sensible lattice operator.

Next let us examine one-loop perturbation theory, taking the vacuum polarization $\Pi_{\mu v}(p)$ as an example. We recall that the interacting theory is defined using the one-component formulation, and that all of its symmetries are needed to ensure that mass terms will always renormalize multiplicatively [2]. How to construct an interacting theory in the taste basis is an issue that we discuss later. For massless staggered fermions one obtains $\Pi_{\mu v}(p)=\left(\delta_{\mu v} p^{2}-p_{\mu} p_{v}\right)(4 c \log (p a)+$ contact terms). The logarithmic term is universal. The coefficient $c$ is the same as one would obtain for a single quark in a continuum calculation, or for that matter, in a lattice calculation using any one-flavor Dirac operator, such as a Wilson fermion. The non-analytic, long-distance part of the vacuum polarization of staggered fermions will therefore be reproduced by (say) four Wilson fermions. Taking the fourth root would replace these four Wilson fermions by one, implying that the fourth root of the long-distance part of the determinant is a sensible quantity.

Like in any perturbative lattice calculation, one-loop diagrams with staggered fermions inside the loop also contain contact terms, which are all local and gauge invariant. The first of those will correspond to (a lattice discretization of) $F_{\mu \nu}^{2}$, and has the effect of a finite renormalization of the coupling constant. Other terms correspond to irrelevant operators. Similar statements apply to a calculation with Wilson fermions. However, the contact terms are not universal, and the staggeredfermions contact terms are not equal to four times the Wilson-fermions contact terms. But then, this short-distance discrepancy is not a problem: In lattice perturbation theory, the fourth root of the staggered determinant will be almost equal to a Wilson-fermion determinant; and, if we wish to, we can always make up for the difference in the contact terms by introducing innocuous, local modifications to the gauge-field action.

The fourth-root trick is also valid within the framework of low-energy effective lagrangians that capture the infra-red limit of QCD [7]. The remaining challenge is to promote the arguments to a fully non-perturbative setup. The lesson is that we will need a device that can separate out long-distance from short-distance physics, thus allowing us to treat differently the two parts of the determinant. This is precisely what Renormalization-Group (RG) blocking was designed for [?, 7 ].

2. Renormalization-group transformations. Our first task is to establish the validity of of the fourthroot trick in the free theory, using RG block transformations [10]. A single RG blocking step works as follows [8]:

$$
\begin{aligned}
Z & =\int d \psi d \bar{\psi} \exp \left(-\bar{\psi} D_{0} \psi\right) \\
& =\int d \psi d \bar{\psi} d q d \bar{q} \exp \left(-\bar{\psi} D_{0} \psi-\alpha\left(\bar{q}-\bar{\psi} Q^{\dagger}\right)(q-Q \psi)\right) \\
& =\operatorname{det}\left(G_{1}^{-1}\right) \int d q d \bar{q} \exp \left(-\bar{q} D_{1} q\right) .
\end{aligned}
$$

The blocking parameter $\alpha$ has mass dimension one. Here $\psi, \bar{\psi}$ live on the original lattice while $q, \bar{q}$ live on the blocked lattice. On the second line, the integrand has been multiplied by one; on 
the last line, the $\psi, \bar{\psi}$-integration has been carried out. The induced operator on the coarse lattice is $D_{1}$, and $G_{1}^{-1}=D_{0}+\alpha Q^{\dagger} Q$. We will assume that the blocking step replaces each $2^{4}$ hypercube by one site of the coarse lattice. The blocking kernel corresponds to the arithmetic mean of $\psi$ over a $2^{4}$ hypercube,

$$
(Q \psi)(\tilde{x})=2^{-4} \sum_{r_{\mu}=0,1} \psi\left(2 \tilde{x}_{\mu}+r_{\mu}\right) .
$$

If we apply the blocking transformation $n$ times, the result is similar to Eq. (3) with $G_{1} \rightarrow G_{n}$, $D_{1} \rightarrow D_{n}$, where (see ref. [10] for further explanations)

$$
G_{n}^{-1}=D_{0}+\alpha_{n} Q_{n}^{\dagger} Q_{n}, \quad D_{n}^{-1}=\alpha_{n}^{-1}+Q_{n} D_{0}^{-1} Q_{n}^{\dagger} .
$$

Here $Q_{n}=Q^{(n)} Q^{(n-1)} \cdots Q^{(1)}$ and $1 / \alpha_{n}=1 / \alpha^{(n)}+2^{-4} / \alpha^{(n-1)}+2^{-8} / \alpha^{(n-2)}+\cdots$, where $Q^{(j)}$ and $\alpha^{(j)}$ are respectively the blocking kernel and parameter used in the $j$-th step.

In order to establish the locality of the fourth root of the free staggered operator we begin with the taste representation (2). The lattice spacing is $2 a$. Applying $n$ blocking transformations, we obtain a coarse-lattice spacing $a_{c}=2^{n+1} a$. The blocking parameter is fixed to be $\alpha^{(j)}=\alpha$ where $\alpha=O\left(1 / a_{c}\right)$, for all steps. We will take the limit $n \rightarrow \infty$ while holding $a_{c}$ fixed. Thus the original lattice spacing vanishes like $2^{-(n+1)}$. For any finite $n$, the generalization of Eq. (3) gives

$$
\operatorname{det}\left(D_{0}\right)=\operatorname{det}\left(G_{n}^{-1}\right) \operatorname{det}\left(D_{n}\right) .
$$

It can be shown [6] that $D_{n}, G_{n}$, and $G_{n}^{-1}$, are all local operators on the coarse lattice, i.e. their kernels decay exponentially with an $O\left(1 / a_{c}\right)$ decay rate, uniformly in $n$. The limit $n \rightarrow \infty$ exists, and we find $\operatorname{det}\left(D_{0}\right)=\operatorname{det}\left(G_{\infty}^{-1}\right) \operatorname{det}\left(D_{\infty}\right)$, in obvious notation.

In the massless case the blocked propagators have the general form

$$
D_{n}^{-1}(p)=\alpha_{n}^{-1}-\sum_{\mu}\left(i\left[\gamma_{\mu} \otimes I\right] \mathscr{A}_{\mu}^{n}(p)+\left[\gamma_{5} \otimes \tau_{5} \tau_{\mu}\right] \mathscr{B}_{\mu}^{n}(p)\right)
$$

where $\alpha_{n}=15 \alpha /\left(16\left(1-2^{-4 n}\right)\right)$. For small $p a_{c}$, one has $\mathscr{A}_{\mu}^{n}(p)=\left(p_{\mu} / p^{2}\right)\left(1+O\left(p a_{c}\right)\right)$. This corresponds to a wave-function renormalization equal to unity, as it must be for a free theory. In units of $a_{c}$, the taste-violating amplitudes $\mathscr{B}_{\mu}^{n}(p)$ scale like $a / a_{c} \propto 2^{-n}$, uniformly in $p$. The taste breaking originates from an irrelevant operator (the last term in Eq. (2)), and this is indeed the scaling anticipated for such an operator. Hence $\mathscr{B}_{\mu}^{\infty}(p)=0$, and $D_{\infty}$ factorizes as $D_{\infty}=D_{\mathrm{rg}} \otimes I$. The fourth root is $\operatorname{det}^{1 / 4}\left(D_{\infty}\right)=\operatorname{det}\left(D_{\mathrm{rg}}\right)$. Being a fixed-point operator (in the massless case), $D_{\mathrm{rg}}$ satisfies the Ginsparg-Wilson relation [11].

The operator $G_{\infty}^{-1}$ has an $O\left(1 / a_{c}\right)$ gap. The Dirac operator $D_{0}$ has a zero at $p=0$ (only) and, for any $n$, this zero is lifted when adding the blocking-kernel part whose maximum is obtained at $p=0$ (see Eq. (Ф)). As a result, there exists a local fourth-root operator $\mathscr{M}$ satisfying $\operatorname{det}^{1 / 4}\left(G_{\infty}^{-1}\right)=$ $\operatorname{det}(\mathscr{M})$ having a similar gap, whose kernel has an $O\left(1 / a_{c}\right)$ decay rate too.

3. Interacting staggered fermions. Writing the interacting, one-component staggered action as $\bar{\chi}\left(D_{s}+m\right) \chi$, the fourth-root trick amounts to the prescription (keeping to a one-flavor theory for notational simplicity)

$$
\begin{aligned}
\langle\chi(x) \bar{\chi}(y) \cdots\rangle & =Z^{-1} \int \mathscr{D} U \operatorname{det}^{1 / 4}\left(D_{s}+m\right) e^{-S_{g}}\left(D_{s}+m\right)^{-1}(x, y) \cdots, \\
Z & =\int \mathscr{D} U \operatorname{det}^{1 / 4}\left(D_{s}+m\right) e^{-S_{g}}
\end{aligned}
$$


An essential element of the RG program is the emergence of the intermediate scale $a_{c}$, which defines the separation between what we consider as short-distance and long-distance physics. In the interacting theory we will again aim at generating through RG blocking a new lattice theory, whose spacing $a_{c}$ satisfies $a \ll a_{c} \ll \Lambda_{Q C D}^{-1}$. The limit $n \rightarrow \infty$ at fixed $a_{c}$ (where $a / a_{c} \rightarrow 0$ ) will again, hopefully, give rise to a local lattice theory on the coarse lattice that, at the same time, will reproduce the physical observables of the original rooted staggered theory. We now list the main issues that arise in the interacting theory. A detailed account will appear elsewhere [12].

- Gauge-field blocking and gauge invariance. The gauge-field blocking can be done in the usual way, see e.g. ref. [9]. The blocking of all fields must respect gauge invariance. For example, suitable parallel transporters [13] must be introduced into the fermion blocking kernel.

- Hypercubic invariance. For blocking of $2^{4}$ hypercubes, it is not possible to construct gaugecovariant blocking kernels that will transform covariantly under hypercubic rotations as well. A projection onto hypercubic invariant observables can nevertheless be enforced. It is equivalent to introducing at each blocking step an action-less local field that lives on the coarse lattice; its (discrete) value at a given hypercube sets the relative coordinates of the site to which we will parallel transport all the variables residing inside that hypercube.

- Observables. The observables of the coarse-lattice theory form a subset of the original observable. The RG transformation(s) define a local mapping of the coarse-lattice observables back into the original fine lattice. This relation is important because, in the absence of a normal path integral, the fourth-root prescription is defined from the outset in terms of observables, cf. Eq. (8) above.

- From one-component to taste. As already mentioned, the interacting theory is originally defined in the one-component formalism. We use the resemblance between Eqs. (11) and (4) to perform a first, special, blocking step. This step realizes a covariant version of Eq. (1), thus defining a tastebasis version of the interacting theory [10, 13]. Since the observables of this interacting theory form a subset of the original observables, in effect all the symmetries of the one-component theory remain intact. The disastrous mass terms, which are generated if one gauges the taste representation in a simple-minded way [14], are avoided.

- "Postponing" multi-fermion interactions. Equation (6) plays the key role of providing the shortdistance - long-distance separation at the level of the fermion determinant. A technical difficulty is that, as soon as we RG block the gauge fields even once, multi-fermion interactions are generated, and any fermionic path integral is no longer a determinant. The simple solution, valid for any finite number of blocking steps, is to perform all the fermion blocking steps ahead of all the gauge-field blocking steps. In this context one can still make use of Eq. (6).

- Scaling. It will be unrealistic to expect rigorous proofs in the interacting case. With all the above elements, what one achieves is a framework for constructing an RG blocked version of the interacting, rooted staggered theory. The familiar scaling behavior, derived using perturbation theory, should then apply for large-enough number of blocking steps $n$, (equivalently, small-enough a). This should imply in particular that all the taste-breaking effects, that always originate from irrelevant operators, will again be damped like $a / a_{c} \propto 2^{-n}$. While not a proof, this suggests that, for $n \rightarrow \infty$, the RG blocked lattice theory emerging from the rooted staggered theory is local.

4. Concluding remarks. An advantage of the RG program is that, since it is formulated in a completely non-perturbative language, it is amenable to numerical tests. Numerical evidence for 
the (approximate) four-fold degeneracy of the low-lying staggered eigenmodes may be found in ref. [15]. For first numerical tests of the scaling properties of the RG blocked fermion propagator in the interacting rooted theory, see ref. [13].

Acknowledgements. I thank Claude Bernard, Carleton Detar, Maarten Golterman and Francesca Maresca for numerous valuable discussions. This work is supported by the Israel Science Foundation under grant no. 222/02-1.

\section{References}

[1] J. B. Kogut and L. Susskind, Hamiltonian Formulation Of Wilson's Lattice Gauge Theories, Phys. Rev. D 11, 395 (1975).

[2] M. F. L. Golterman and J. Smit, Selfenergy And Flavor Interpretation Of Staggered Fermions, Nucl. Phys. B 245, 61 (1984).

[3] E. Marinari, G. Parisi and C. Rebbi, Monte Carlo Simulation Of The Massive Schwinger Model, Nucl. Phys. B 190, 734 (1981).

[4] C. T. H. Davies et al. [HPQCD Collaboration], High-precision lattice QCD confronts experiment, Phys. Rev. Lett. 92, 022001 (2004) [hep-lat/ 030400 4]; C. Aubin et al. [MILC Collaboration], Light pseudoscalar decay constants, quark masses, and low energy constants from three-flavor lattice QCD, Phys. Rev. D 70 (2004) 114501 [hep-lat / 0407028 ].

[5] F. Gliozzi, Spinor Algebra Of The One Component Lattice Fermions, Nucl. Phys. B 204, 419 (1982); A. Duncan, R. Roskies and H. Vaidya, Monte Carlo Study Of Long Range Chiral Structure In QCD, Phys. Lett. B 114, 439 (1982); H. Kluberg-Stern, A. Morel, O. Napoly and B. Petersson, Flavors Of Lagrangian Susskind Fermions, Nucl. Phys. B 220, 447 (1983).

[6] T. Balaban, M. O'Carroll and R. Schor, Block Renormalization Group For Euclidean Fermions, Commun. Math. Phys. 122, 233 (1989);

[7] C. W. Bernard and M. F. L. Golterman, Partially quenched gauge theories and an application to staggered fermions, Phys. Rev. D 49, 486 (1994) [hep-lat/9306005].

[8] T.L. Bell and K.G. Wilson, Finite-lattice approximations to renormalization groups, Phys. Rev. B 11, 3431 (1975).

[9] For a review, see: P. Hasenfratz, The theoretical background and properties of perfect actions, hep-lat/9803027.

[10] Y. Shamir, Locality of the fourth root of the staggered-fermion determinant: Renormalization-group approach, Phys. Rev. D 71, 034509 (2005) [hep-lat / 0412014$].$

[11] P. H. Ginsparg and K. G. Wilson, A Remnant Of Chiral Symmetry On The Lattice, Phys. Rev. D 25, 2649 (1982); F. Maresca and M. Peardon, A path-integral representation of the free one-flavour staggered-fermion determinant, hep-lat/0411029; see also ref. [?].

[12] Y. Shamir, work in progress.

[13] F. Maresca [MILC Collaboration], these proceedings.

[14] P. Mitra and P. Weisz, On Bare And Induced Masses Of Susskind Fermions, Phys. Lett. B 126, 355 (1983).

[15] E. Follana, A. Hart, C. T. H. Davies and Q. Mason [HPQCD Collaboration], The low-lying Dirac spectrum of staggered quarks, hep-lat/0507011, and references therein. 